\title{
COVID-19 and Autism - Part 2
}

\author{
Gary Steinman* and David Mankuta \\ Dept. of Obstetrics \& Gynecology, Hadassah Hospital - Hebrew University, Ein Kerem, Jerusalem 12000, Israel
}

${ }^{\star}$ Corresponding author: Gary Steinman, Dept. of Obstetrics \& Gynecology, Hadassah Hospital - Hebrew University, Ein Kerem, Jerusalem 12000, Israel; E-mail: dav4601@aol.com

Received: December 16, 2020; Accepted: December 27, 2020; Published: January 03, 2021

\begin{abstract}
When severe cases of febrile viral infections occur in pregnant women, there is an increased risk of autism in the offspring a year or two after the birth. It would seem that the primary reason for this is increased levels of blood pro-inflammatory cytokines and unenhanced amounts of IL10, an antiinflammatory interleukin. Most important is the decreased concentration of insulin-like growth factor-1, which slows the myelination of new neurons, causing dysconnectivity of cerebral nerve circuits.
\end{abstract}

\section{Introduction}

In the preceding parts of this report [1,2], the apparent relationship between severe maternal inflammatory disease during pregnancy (e.g., COVID-19) and the increased incidence in their children's autism was discussed. Under these conditions, the need for insulin-like growth factor (IGF1) to promote the myelination of new nerves in the fetus was emphasized. The postpartum persistence of an IGF1 deficiency could lead to the development of brain dysconnectivity and autistic behavior in the neonate at age 1-2 years. Plausible etiologies of this in the baby will be considered here. Central to this phenomenon is the important rise in the biosynthesis of interleukins. They are a part of the immune system and are synthesized by lymphocytes, monocytes, macrophages, and endothelial cells. Cytokines include lymphokines, interferons, tumor necrosis factors, interleukins, and chemokines [3-5].

The involvement of Interleukins (IL) in cellular functions can be divided into two main groups:

Th1 - units that promote cell-mediated immune response and the production of IFNg, IL-2, and TNF-b;

Th2 - units that possess various cytokines including IL-4, IL-5, IL-6, IL-9, IL-10, and IL-13. An imbalance between these two main groups can possibly lead into the pathogenesis of autism.

It can be noted for the healthy neonate that in the period between birth and the first year or two of extrauterine life, the accumulation of various cytokines continues independently on the course preset by the balance established before and at birth. This condition apparently involves the equilibrium between IL-10 and the pro inflammatory interleukins primarily. Direct maternal physical influences on the baby's neural health terminate at birth. The child's potential neurologic status will be determined by ante- and postpartum genetic and environmental factors, especially the capacity to synthesize enough IGF1 to establish a functional, healthy neurologic milieu autonomously [6].

\section{Autism Initiation - Phase \# 1 (Prenatal)}

It would appear that the generation of autism begins with the intrauterine fetus exposed before birth to elevated maternal temperatures (e.g., caused by COVID-19, influenza, SARS-CoV, $\mathrm{H} 5 \mathrm{~N} 1$, or MERS-CoV infection) and groups of interleukins due to the disease. On the one hand, myelination of new fetal nerves is primarily dependent on the presence of sufficient IGF1. In the developing baby, ante- and postpartum rising interleukin levels are matched with falling IGF1. In laboratory animals, a link has been demonstrated between maternal immune activation and autism-like outcomes. In a post-mortem study of human brains, elevated cytokines and infection states have been observed. Children with inflammatory diseases typically have reduced IGF1 and elevated IL-6 [1,2]. Overall, a balance between pro-inflammatory and anti-inflammatory functional cytokines is needed for good health. Antepartum maternal infection can promote the release of specific cytokines such as IL6 into the mother's bloodstream. In a meta-analysis of $>40,000$ autism cases, maternal infection during pregnancy was correlated with autism in the babies. In contrast, IL-10 is anti-inflammatory. In a study of 69 severe type COVID-19 human patients where IL- 6 was used as a monitoring marker, elevated levels of $\mathrm{LDH}, \mathrm{C}$-reactive protein, ferritin, and D-dimer were commonly found. In IL10-deficient mice, inflammatory bowel disease is enhanced. Over-secretion of cytokines, especially IL-6, is a sign warning of a possible "cytokine storm". Typically, the cytokine, IL-10, offsets the increase of IL-6 [7-12]. In a study of 538 autistic children versus 421 typically developing controls, the risk of autism from fever in the gravidas was attenuated among mothers who used antipyretics [13].

\section{Autism Promotion - Phase \#2 (Birth To 1-2 Years)}

In this covert phase, the classical characteristics of autism have not yet emerged, but quantitative changes in the underlying cytokines are progressing. Bioactive cytokines appear to participate in the resistance to or involvement in the development of autism. For example, the pro- 
inflammatory group would include IL-1a, IL-6, IL-8, and IL-17, in contradistinction to IL-10. In a situation where the release of IL-6 is gradually enhanced, the production of IGF1 is reduced. A safeguard for this is the counterpart release of IL-10. However, if the baby has already developed autistic tendencies from intrauterine exposure to pathologic febrile conditions (e.g., maternal COVID-19), the production of IL10 do not increase and the amount of IGF1 would fall. With sufficient decline, developmental phase $\# 2$ will be nearing its completion, and phase \#3, with overt manifestations of autism, will begin.

In a study with laboratory mice, injection of IGF1 decreased vascular expression of the cytokines IL- 6 and TNFa. In other words, circulating IGF1 apparently decreases inciting reactions. This is in combination with the role of IGF1 in promoting essential myelination in new nerves to accelerate the transmission of commands for bodily actions. As a result, the preliminary circuits created in the newborn are fixed in place for longterm function. Without this, nervous pathways, especially in the brain, would be of reduced utility, accuracy, and velocity. Grossly autistic behavior is typically restrained before age 1 year. This would suggest that altered neurogenesis due to IGF1 deficiency is continuing in the growing baby between birth and 1 year without noticeable external factors effecting these changes in most cases. In transgenic mice, it was found that increased serum IL-6 was associated with low serum IGF1 levels and growth delay. A typical precursor of autism in humans is the finding of cytokines TNFa, IL-6, IL-1b in the fetus's brain or liver, whereby the IGF1 level would be insufficient. In addition to promoting myelination of nerves, IGF1 reduces provocative responses and suppresses oxidative stress, risk of autism, and atherosclerosis progression.

If a developing fetus is exposed continuously to increased levels of IL- 6 and reduced IGF1 during gestation, the neonate is at increased risk for affected cognition by 12 months old and altered brain architecture, executive function, behavior, and working memory at 2 years of age [14-17].

\section{Autism Persistence - Phase \#3 (Age >2 Years)}

In this overt phase in particular, bioactive cytokines seem to participate in the resistance to or participation in the development of autism. As noted above, functional interleukins are typically divided into two opposing groups. For example, the first group would typically include IL-1a, IL-6, IL-8, and IL-17, and the second, IL-10. In a situation where the release of IL- 6 is gradually enhanced, the production of IGF1 is reduced, as noted earlier. A safeguard for this in an unaffected child is the analogous release of IL-10. However, if the baby has already developed autistic tendencies from intrauterine exposure to provoking conditions (e.g., fever due to maternal COVID-19), the production of IL-10 would not be increased and the amount of IGF1 would fall. In another study, children aged 3-11 years who were diagnosed as autisticwere tested for cytokine status. Interleukin groups Th1 and Th2 were found to be elevated in the blood of autistic children above unaffected controls, whereas the concentration of IL-10 displayed no compensatory increase between the two groups. In a further observation of children in ages 2-5 years, the levels of interleukins IL-1b and IL-6 in autistic youngsters were twice those of normally developing children. Elevation of IL-6 in humans with autism is a common finding. In a meta-analysis with
743 autistic participants and 592 healthy controls, quantities of IL$1 \mathrm{~b}$, IL-6, and IL-8 were significantly higher in affected individuals. No difference was found between the two groups tested for 12 other common cytokines. Also, the cerebrospinal fluid from autistic patients revealed increased IL-6, IL-8, and IFNg. The postmortem examination of autistic human brains typically exposed marked over-production of IL-1b, IL-6, IL-17, and TNFa. Mice with elevated brain IL-6 display alterations in excitatory/inhibitory synaptic transmissions [18-30].

\section{Conclusions}

Increasing attention is being given to the employment of IGF1 as a means for attenuating or preventing autism [31,32]. For example, in reference \#1 in this report, the proposed use of breast feeding to replace deficient IGF1 in babies before symptoms of autism appear is advocated. Recently conveyed data indicate that coronavirus in the mother does not ascend into the breast milk during pregnancy [33]. The neonatal goal is to prevent the production of insufficiently myelinated neo-neurons which could result in brain dysconnectivity in the infant. Alternatively, modifications of the IGF1 polypeptide for use in ameliorating autism-like conditions such as Phelan-McDermid and Rett Syndrome have been tested [31,34].

The data presented here clearly demonstrate the participation of pro-inflammatory cytokines in the generation of autism. In many cases this is due to a febrile process in the gravid mother. Fever together with pro-inflammatory interleukins is an apparent factor promoting autism increase in the neonate. It remains to be determined if this is only in some, most, or all cases of autism.

\section{Declaration of Competing Interests}

The authors declare that they have no known competing financial interests or personal relationships that could have appeared to influence the work reported in this paper.

\section{Acknowledgements}

The authors wish to thank Roberta Zuckerman for her helpful discussions about the presentation of this communication, as well as Aviva Adler, librarian, for her cooperative assistance in locating relevant literature references.

\section{References}

1. Steinman G (2020) Covid-19 and autism. Med Hypoth 142: 109797. [crossref]

2. Steinman G (2020) Febrile coronavirus and autism. J Endo Metab Res 1: 1-7.

3. Mosaad M, Nasralah M, Quinn T, et al. (2014) Inflammation In The cause of autism. G. Steinman, (eds.), Baffin Books Pub, New York, chap. 6C.

4. Wei H, Chadman KK, McCloskey DP, Ashfaq M Sheikh, Mazhar Malik, et al. (2012) Brain IL-6 elevation causes neuronal circuitry imbalances and mediates autism-like behaviors. Biochim Biophys Acta 1822: 831-842. [crossref]

5. Bilbo SD, Black CL, Bolton JL, Richa Hanamsagar, Phuong K Tran (2018) Beyond infection - maternal immune activation by environmental factors, microglial development, and relevance for autism spectrum disorders. Exp Neurol 299: 241-251. [crossref]

6. Iyer SS, Cheng G (2012) Role of Interleukin 10 transcriptional regulation in inflammation and autoimmune disease. Crit Rev Immunol 32: 23-63. [crossref]

7. Hempel L, Korholz D, Bonig H, A Klein-Vehne, J Packeisen, et al. (1995) Interleukin-10 directly inhibits the interleukin-6 production in T-cells. Scand J Immunol 41: 462-466. [crossref] 
8. Moore KW, Malefyt RdW, Coffman RL, et al. (2001) Interleukin-10 and the interleukin-10 receptor. Annu Rev Immuno 19: 683-765. [crossref]

9. Ruan Q, Yang K, Wang W (2020) Clinical predictors of mortality due to Covid-19 based on an analysis of data of 150 patients from Wuhan, China. Inten Care Med 46: 846-848. [crossref]

10. Strober W, Fuss IJ (2011) Proinflammatory cytokines in the pathogenesis of inflammatory bowel diseases. Gastroent 140: 1756-1767. [crossref]

11. Liu T, Zhang J, Yang Y (2019) The potential role of IL-6 in monitoring coronavirus disease 2019. The Lancet D-20-02786.

12. Masi A, Glozier N, Dale R (2017) The immune system, cytokines, and biomarkers in autism spectrum disorder. Neurosci Bull Doi: 10.1007/s12264-017-0103-8. [crossref]

13. Zerbo O, Iosif A, Walker C (2013) Is maternal influenza or fever during pregnancy associated with autism or developmental delays? Results from the CHARGE Study. J Autism Dev Disord 43: 25-33. [crossref]

14. Cirillo F, Lazzeroni P, Sartori C (2017) Inflammatory diseases and growth: Effects on the GH-IGF axis and on growth plate. Int J Mol Sci 18: 1878-1887. [crossref]

15. Martins-Filho PR, Tanajura DM (2020) Covid-19 during pregnancy: Potential risk for neurodevelopmental disorders in neonates? Europ J Obs Gyn Reprod Biol 250: 255-256. [crossref]

16. Sukhanov S, Higashi Y, Shai S-Y (2007) IGF-1 reduces inflammatory responses, suppresses oxidative stress, and decreases atherosclerosis progression in apoEdeficient mice. Artioscler Thromb Vasc Biol Doi: 10.1161/ATVBAHA.107.156257. [crossref]

17. Yoshikawa T, Hill T, Li K (2009) Severe acute respiratory syndrome (SARS) corononavirus-induced lung epithelial cytokines exacerbate SARS pathogenesis by modulating intrinsic functins of monocyte-derived macrophages and dendritic cells. J Virol 83: 3039-3048. [crossref]

18. Ashwood P, Krakowiak P, Picciotto IH (2011) Associations of impaired behaviors with elevated plasma chemokines in autism spectrum disorders. J Neuroimmunol 232: 196-199. [crossref]

19. Vargas DL, Nascimbene C, Kishnan C (2005) Neuroglial activation and neuroinflammation in the brain of patients with autism. Ann Neurol 57: 67-81. [crossref]

20. Siniscalco D, Schultz S, Brigida A, et al. (2018) Inflammation and neuro-immune dysregulations in autism spectrum disorders. Pharm 11: 56-69. [crossref]
21. Patterson PH (2011) Maternal infection and immune involvement in autism. Trends Mol Med 17: 389-394. [crossref]

22. Jacome MCI, Chacon LMM, Cuesta HV (2016) Peripheral inflammatory markers contributing to comorbidities in autism. Behav Sci (Basel) 6: 29. [crossref]

23. Ashwood P, Krakowiak P, Hertz-Picciotto I (2011) Elevated plasma cytokines in autism spectrum disorders provide evidence of immune dysfunction and are associated with impaired behavioral outcome. Brain Behav Immun 25: 40-45. [crossref]

24. Zie J, Huang L, Xiaohong L, et al. (2017) Immunological cytokine profiling identifies TNF-a as a key molecule dysregulated in autistic children. Oncotarget 8: 8239082398. [crossref]

25. Turner MD, Nedjai B, Hurst T, et al. (2014) Cytokines and chemokines: At the rossroads of cell signalling and inflammatory disease. Biochim Biophys Acta 1843: 2563-2582. [crossref]

26. Pardo CA, Vargas DL, Zimmerman AW (2005) Immunity neuroglia and neuroInflammation in autism. Int Rev Psychiatry 71: 485-495. [crossref]

27. Molloy CA, Morrow AL, Meinzen-Derr J (2006) Elevated cytokine levels in children with autism spectrum disorder. J Neuroimmun 172: 198-205. [crossref]

28. MontanoMendoza VM (2020) Interleukin-17: A potential therapeutic target in Covid-19. J Infect 81: e136-138. [crossref]

29. Lima MES, Barros LCM, Aragao GF (2020) Could autism spectrum disorders be a risk factor for Covid-19? Med Hypoth 144: 109899. [crossref]

30. Raucci F, Mansour AA, Casillo GM, et al. (2020) Interleukine-17A (IL-17A), a key molecule of innate and adaptive immunity, and its potential involved in Covid-19related thrombotic and vascular mechanisms. Autoimmun Rev 17: 102572. [crossref]

31. Steinman G, Mankuta D (2020) The role of oligopeptides in preventing autism. Med Hypoth 138: 109604.

32. Steinman G, Mankuta D (2019) Molecular biology of autism's etiology - an alternative mechanism. Med Hypoth 130: 109272. [crossref]

33. Dumitriu D, Emeruwa UN, Hanft E (2020) Outcomes of neonates born to mothers with severe acute respiratory syndrome coronavirus 2 infection at a large medical center in NYC. JAMA Ped doi: 10.1001/jamapediatrics.2020.4298. [crossref]

34. Linker SB, Mendes APD, Marchetto MD (2020) IGF-1 treatment causes unique transcriptional response in neurons from individuals with idiopathic autism. Molec Autism 11: 55-67. [crossref]

\section{Citation:}

